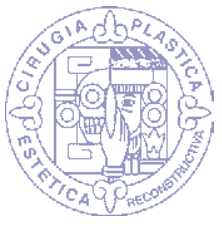

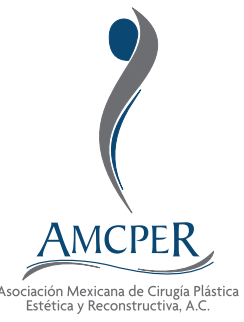

\title{
Abdominoplastia más cesárea en el mismo tiempo quirúrgico, 25 años de experiencia
}

\author{
Abdominoplasty plus caesarean section at the same \\ surgical time, 25 years of experience
Dr. Jaime González-Mendoza,* Dr. Francisco Enrique Cerecero-García, Dra. Patricia García-Treviño, ${ }^{\S}$ Dr. Fernando Ramírez Macías-Valadez"

Palabras clave: Abdominoplastia, lipoaspiración, cesárea, salpingoclasia, procedimientos combinados.

Keywords: Abdominoplasty, liposuction, caesarean section, salpingoclasia, combined procedures.

* Cirujano plástico, socio activo de la AMCPER, certificación vigente 733 CMCPER, presidente fundador y miembro activo CCPERA, miembro activo FILACP, ASPS, ISAPS e IMCAS, director y propietario de Spaplastic, S.A. de C.V.

¥ Anestesiólogo

cardiovascular: UANL.

Miembro CMA; CMAA;

COMEXANE.

$\S$ Médico cirujano

partero. Certificado por CONAMEGE. Dermocosmética por la Universidad de Barcelona.

" Médico cirujano.

Certificado por CONAMEGE. Miembro

SNMG, A.C.

Los autores de este artículo no tienen conflicto de intereses que declarar.

Recibido:

25 abril 2019 Aceptado para publicar: 10 agosto 2019

\section{RESUMEN}

La abdominoplastia-cesárea es un procedimiento quirúrgico solicitado ocasionalmente por pacientes que desean que en una sola intervención y convalecencia se efectúen ambos procedimientos para evitar un segundo riesgo quirúrgico y periodo de recuperación, lograr el alumbramiento de su producto y eliminar las secuelas propias de uno o varios embarazos previos. En este trabajo presentamos los resultados de 140 pacientes intervenidas en un periodo de 25 años, observando que las complicaciones detectadas son similares en las pacientes no embrazadas, con resultados estéticos no ideales, pero satisfactorios y ausencia de complicaciones severas o riesgos a la salud o muerte de las pacientes. Concluimos que la combinación de estas cirugías no es lo ideal en tiempo y forma; no obstante, no hay una justificación absoluta que limite su realización.

\section{ABSTRACT}

Abdominoplasty-caesarean section is a surgical procedure requested occasionally by patients who want both procedures with a single intervention and convalescence, to avoid a second surgical risk and recovery period, to achieve the delivery of their baby and eliminate the sequelae of one or several previous pregnancies. In this paper we present the results of 140 patients operated on in a 25-year period, observing that the complications presented are similar to non-pregnant patients, with aesthetic results not ideal but satisfactory and absence of severe complications or risks to the health or death of the patients We conclude that the combination of these surgeries is not ideal in time and form; however, there is no absolute justification that limits their performance.

\section{INTRODUCCIÓN}

S egún las estadísticas de International Society of Aesthetic Plastic Surgery (ISAPS), en el año 2017 se realizaron 802,234 abdominoplastias en el mundo y en México $36,386 .^{1} \mathrm{La}$ abdominoplastia es el procedimiento estético con el mayor número de complicaciones cuya incidencia aumenta cuando se combina con liposucción o algún otro tipo de procedimiento; la complicación más severa es la trombosis. ${ }^{2-10}$

La mujer presenta cambios en todos sus órganos y sus funciones durante el embarazo. Existe un incremento en el fibrinógeno y en los factores VII, VIII, X y XII, estos cambios representan una protección contra la hemorragia al momento del parto, pero también conllevan el riesgo de trombosis. ${ }^{11-14}$

Durante el embarazo se produce flacidez cutánea con formación de pliegues y la pared muscular se debilita, facilitando la protrusión de las vísceras intraabdominales. Algunos cirujanos realizan una abdominoplastia al mismo tiempo que la cesárea con la finalidad de corregir los defectos cutáneos y de la pared muscular y así evitan una cirugía varios meses o años después. Son escasas las publicaciones que existen sobre el tema y son controvertidas. Algunos autores consideran que es un procedimiento seguro y que se pueden obtener buenos resultados; mientras que otros opinan que el aumento del útero y las condiciones de los tejidos dificulta al cirujano estimar adecuadamente la resección cu- 


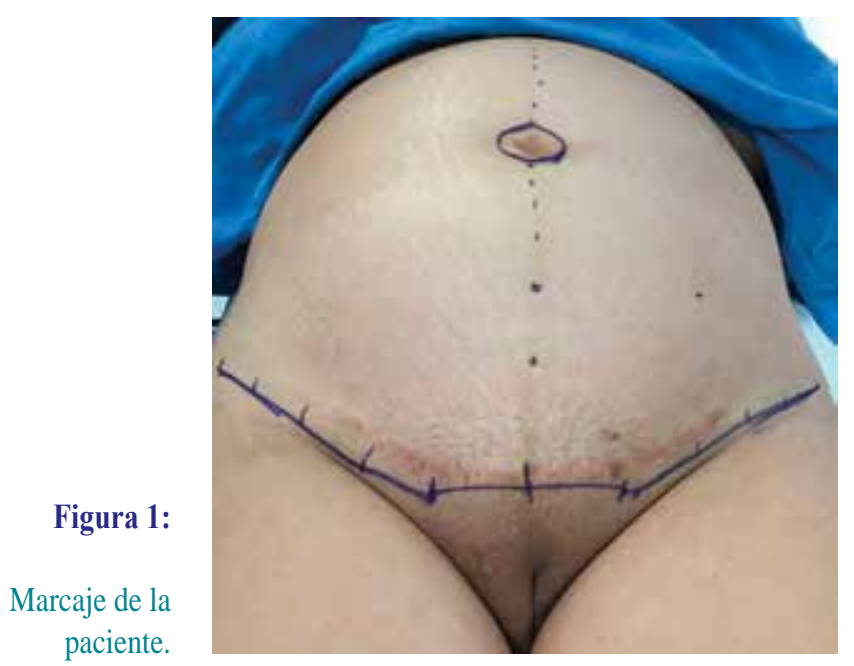

tánea necesaria; además, han reportado una alta incidencia de complicaciones como infección, dehiscencia de herida, necrosis y trombosis. ${ }^{14-18}$

El objetivo del presente trabajo es mostrar los resultados obtenidos al realizar abdominoplastia combinada con cesárea en el mismo tiempo quirúrgico, así como de los cuidados preventivos que hemos implementado para abatir las complicaciones.

\section{PACIENTES Y MÉTODO}

En el periodo comprendido de 1994 a 2019 (25 años), atendimos a 140 mujeres embarazadas, de 25 a 40 años de edad y paridad satisfecha, a quienes realizamos cesárea y abdominoplastia y, en algunos casos, salpingoclasia y liposucción de flancos. Todas las pacientes fueron atendidas en un hospital privado: 126 (90\%) por el mismo equipo médico (cirujano plástico, ginecólogo, anestesiólogo, pediatra y dos ayudantes quirúrgicos) y 14 (10\%) operadas por otro equipo quirúrgico con los mismos criterios establecidos.

Los criterios de inclusión fueron: ser mujer embarazada en perfectas condiciones de salud, con producto único vivo de 36 a 41 semanas de edad gestacional; con índice de masa corporal promedio de 25, con alteraciones en la estética abdominal por haber sobrepasado 11 kg de peso durante la gestación, presencia de estrías, diástasis de rectos abdominales, hernia umbilical, ptosis abdominal, así como valoración y seguimiento mensual de la gestación por parte de su ginecólogo. Pacientes sin riesgos detectados, con ultrasonidos y exámenes de laboratorio seriados sin alteraciones, repetidos 15 días previos a la cirugía, en quienes se descartó infección de vías urinarias, sida, anemia y alteraciones de la coagulación.

Los criterios de exclusión fueron: que las pacientes tuvieran alguna alteración en la glucosa, hipertensión arterial, anemia o cualquier otra morbilidad, así como embarazo con probables alteraciones del producto y/o riesgo de muerte al nacimiento y mujeres programadas para procedimientos combinados que en el momento de la cesárea presentaran hemorragia.

Marcaje. Se realiza siempre con la paciente de pie con rectificación en posición de decúbito dorsal; inicialmente en la zona central, de 5 a $7 \mathrm{~cm}$ de la comisura vulvar superior, con una extensión de 6 a $8 \mathrm{~cm}$ de longitud transversa, iniciando el ascenso lateral bilateral de 10 a 15 $\mathrm{cm}$ de largo dependiendo de cada paciente en particular, sin exceder más de $10 \mathrm{~cm}$ de altura del pliegue inguinal (Figura 1).

Cuidados preoperatorios. Colocación de sonda Foley y venoclisis con catéter $2.1 \times 45 \mathrm{~mm}$; perfusión endovenosa con solución Hartmann; control estricto de uresis y balance de líquidos; colocación de medias de compresión alta (del pie a la ingle), con uso indicado por 15 días y retiro por 30 minutos al día a la hora de la ducha.

Anestesia. En todas las pacientes utilizamos bloqueo regional subaracnoideo con lidocaína y morfina; después del alumbramiento se combinó con sedación intravenosa con midazolam y fentanil. 
Cuidados transoperatorios. Temperatura ambiental del quirófano a $23{ }^{\circ} \mathrm{C}$; antibiótico profiláctico con cefalotina $1 \mathrm{~g}$ IV cada 12 horas hasta completar siete días; etamsilato $4 \mathrm{mg}$ dos ampolletas en la solución base al inicio de la cirugía para evitar el sangrado transquirúrgico y postquirúrgico.

\section{Técnica quirúrgica}

Procedimientos obstétricos. El equipo quirúrgico de ginecología y obstetricia inicia la cirugía, tiene la opción de realizar la incisión horizontal tipo pfannenstiel o vertical en la línea media, ambas concluyen con cesárea tipo Kerr, provocando el alumbramiento del producto y limpieza de cavidad uterina en forma mecánica, seguido de salpingoclasia bilateral, hemostasia de vasos sangrantes, histerorrafia y cierre por planos hasta aponeurosis (Figura 2).

Procedimientos de cirugía plástica. Se procede a realizar liposucción súper húmeda de ambos flancos. Infiltración de solución de Klein modificada (solución salina al 0.9\%: 1,000 mL + dos ampolletas de epinefrina), espera de un tiempo de latencia de 10 a 15 minutos; aspiración de grasa excedente de los costados con cánula de aspiración de 3 a 4 mm, extrayendo no más de 1,000 mL por cada lado (Figura 3). Se continúa con la incisión de la piel previamente marcada y despegamiento del colgajo en plano supraaponeurótico hasta el borde costal, laterales y apófisis xifoides del área central y cauterización de vasos sangrantes. En los casos con diástasis de los músculos rectos anteriores se procede a realizar marcaje con violeta de genciana y afrontar los músculos con puntos simples separados con Vicryl número 1; en segundo plano un surgete continuo en todo el trayecto de la plicatura con Nylon 1. Si existe hernia umbilical, se realiza hernioplastia recolocando el ombligo en su sitio y fijación a la aponeurosis (Figuras 4 a 6). Se coloca a la paciente en posición de Flex $\left(45^{\circ}\right)$ para lograr mejor ajuste del colgajo, evi-
Figura 2:

Salpingoclasia e histerorrafia.
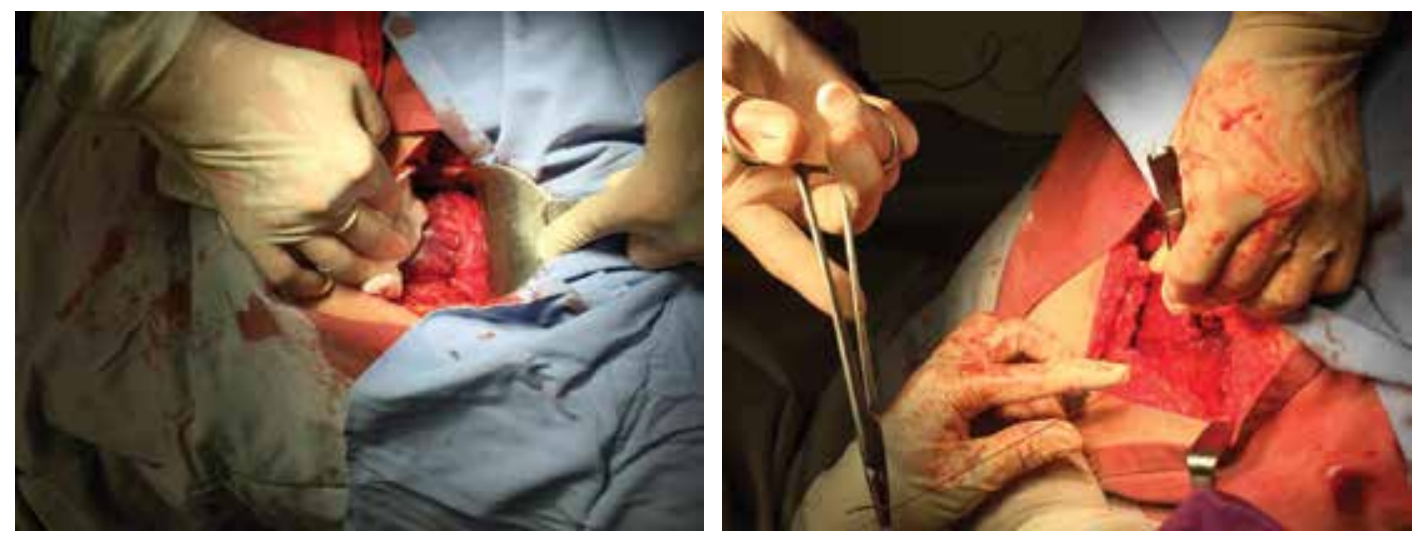

Figura 3:

Liposucción de no más de 1,000 mL.
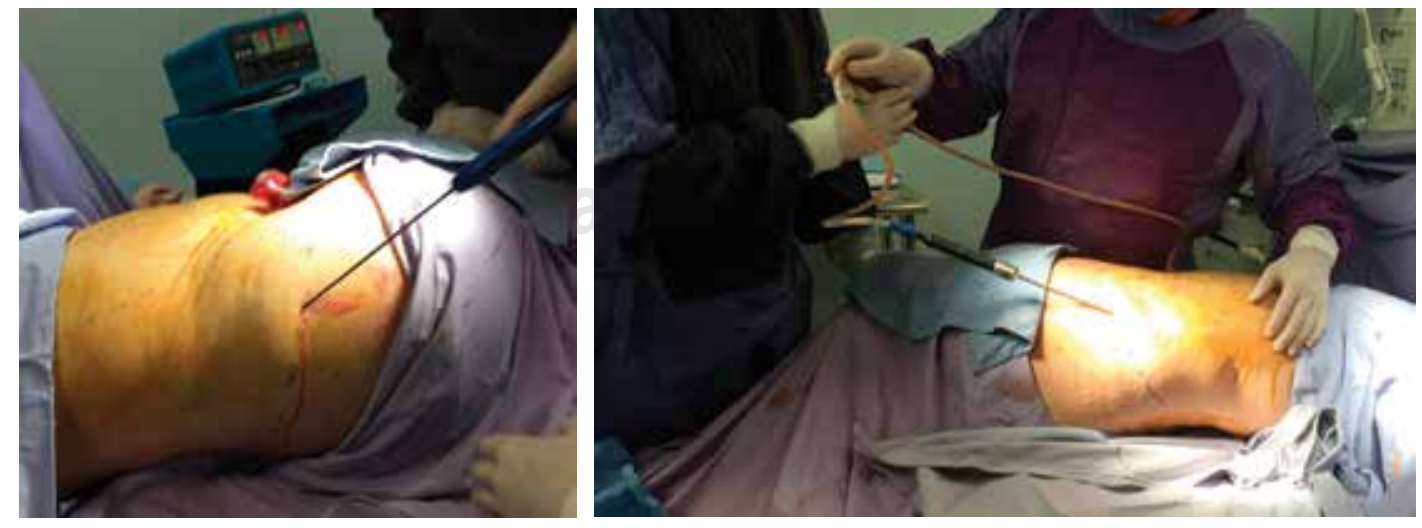
Figura 4:

Inicio de incisiones y levantamiento del colgajo central.
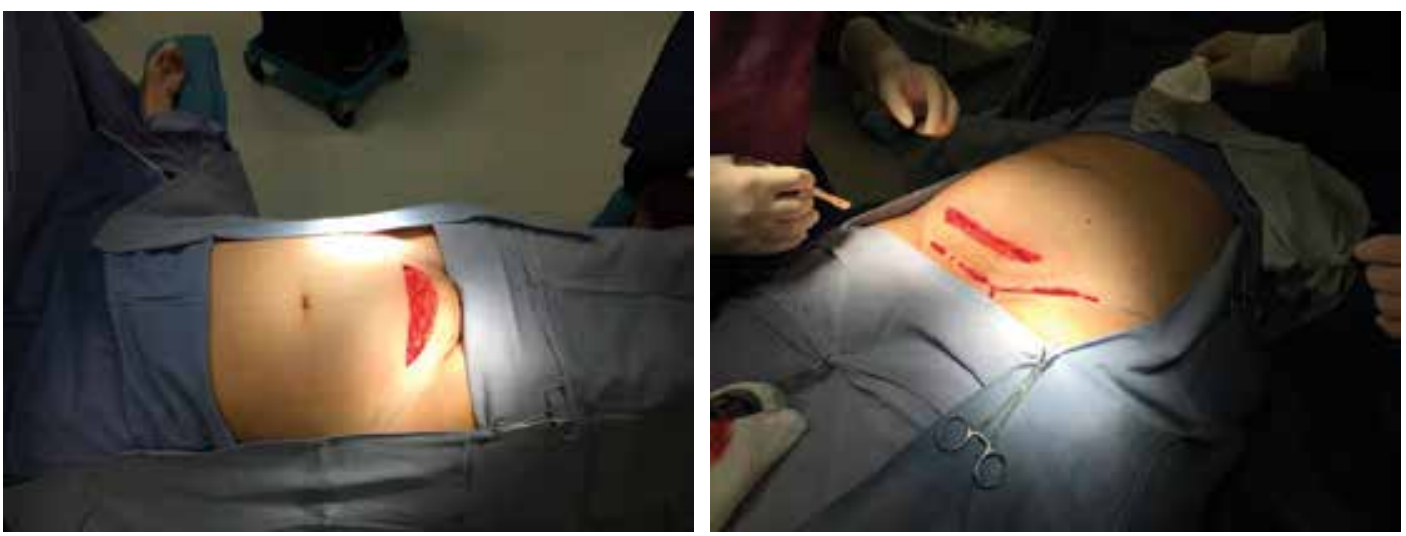

Figura 5:

Marcaje y cierre aponeurótico.
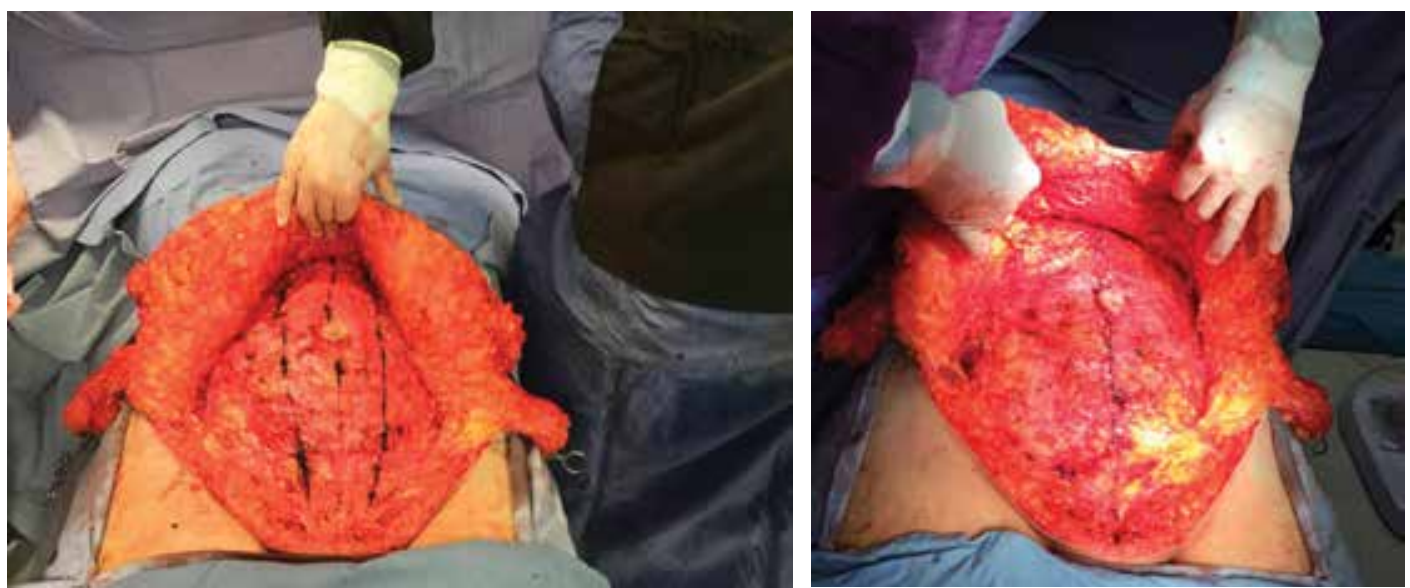

tando la tensión. Se dibuja el excedente cutáneo previo a su resección (Figura 7), localizando el ombligo y por medio de incisión helicoidal de la piel se extrae para su fijación cutánea. Se procede a cauterizar los vasos sangrantes del borde de la herida y se aplican dos tubos de drenaje de $1 / 4^{\prime \prime}$ en la región púbica y se cierra en tres planos (Figura 8): el primer plano de la grasa profunda del colgajo con Vicryl 0 puntos simples, el segundo plano con Vicryl 00 para afrontar el plano superficial graso con puntos simples separados y por último el tercer plano con Nylon 00 puntos continuos intradérmicos en toda la extensión de la herida. Para terminar se coloca vendaje compresivo. El segmento dermoadiposo resecado en dos pacientes fue enviado al laboratorio de patología para estudio histopatológico.

Cuidados postoperatorios. Enoxaparina 30 mg subcutánea cada 12 horas durante 48 horas, iniciando ocho horas después del evento quirúrgico. Analgesia postquirúrgica a base de ketorolaco $30 \mathrm{mg}$ IV cada ocho horas. Ondasetrón 8 mg IV en el transoperatorio para evitar náuseas y vómitos, combinado con ranitidina 150 mg IV. Deambulación temprana, baño y colocación de prenda de compresión de uso

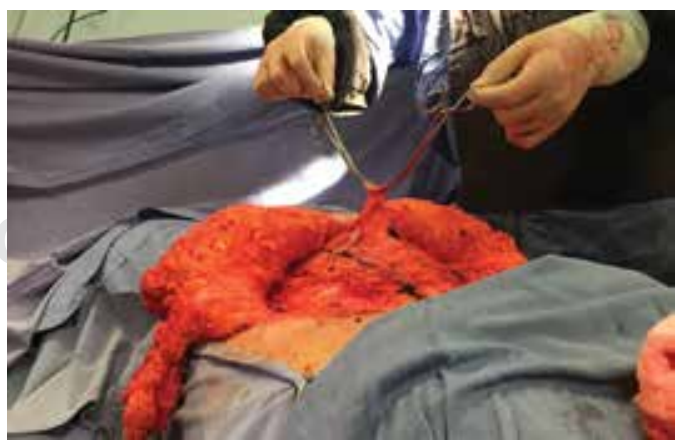

Figura 6: Plastia de hernia umbilical y fijación a la aponeurosis. 


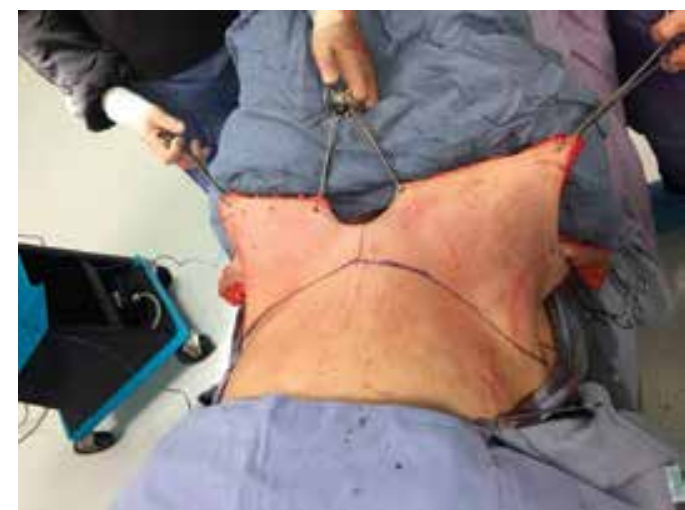

Figura 7: Marcaje y ajuste del colgajo.

las 24 horas por dos meses, con retiro de la misma sólo para el baño diario por 30 minutos.

En el postoperatorio inmediato se lleva a cabo un monitoreo estricto de sus constantes vitales por 48 horas, tratando de mantener una uresis horaria mayor de $50 \mathrm{~mL}$ por hora y una tensión arterial diastólica mayor de $60 \mathrm{mmHg}$. Cuando las cifras son menores, se procede a aplicar solución Hartmann 500 mL en cargas para una hora. Se solicita biometría hemática para valorar la pérdida sanguínea. En caso de cifras de hemoglobina $<$ de $9.5 \mathrm{~g} / \mathrm{dL}$ se procede a transfundir paquete globular. Se mantiene a la paciente en posición estricta de Flex a 45 grados. El egreso hospitalario se efectúa de 48 a 60 horas después de la cirugía, retirando en ese momento los drenes y la sonda Foley.

Al egreso se dan indicaciones domiciliarias: levofloxacino $750 \mathrm{mg}$ vía oral cada 24 horas durante siete días, dexketoprofeno $25 \mathrm{mg}$ vía oral cada seis horas durante siete días, ácido acetilsalicílico 100 mg cada 24 horas, ácido fólico y fumarato ferroso vía oral por 15 días. Uso de medias elásticas las 24 horas durante dos semanas, baño diario con agua a temperatura tibia a fría a tolerancia. La paciente deberá estar siempre sentada y acompañada de un familiar o personal de enfermería. Por 15 días se indica deambulación asistida y dormir en decúbito dorsal en posición de Flex (45). Dieta especial por dos meses, beber de cuatro a cinco litros de líquidos al día, aumentar la ingesta de alimentos que favorecen el tránsito intestinal y eliminar por completo todo tipo de alimentos productores de gases intestinales.
La valoración postoperatoria se efectúa en el consultorio a los 15 días del postoperatorio. En este tiempo se procede al retiro de puntos y se elimina la postura de Flex. Se realiza la exploración física buscando la presencia de seromas, compromiso vascular cutáneo, dehiscencia de herida y/o infección. De encontrar alguna complicación se determinan los procedimientos correctivos adecuados a los hallazgos.

En los casos de seroma postquirúrgico, se tratan con punciones infraumbilicales con catéter intravascular calibre 14GA $2.1 \times 45 \mathrm{~mm}$, apoyado por succión negativa con jeringa de 20 $\mathrm{mL}$ hasta la extracción completa. Las punciones se repiten cada tercer día hasta la resolución.

Aspecto bioéticos. Es un estudio retrospectivo de pacientes operadas por el autor, por lo que no se requiere de un protocolo autorizado por algún comité de investigación y ética. Los datos fueron obtenidos de los expedientes clínicos y del archivo fotográfico. Todas las pacientes firmaron carta de consentimiento informado autorizando al equipo quirúrgico a realizar los procedimientos. Los datos personales son manejados en forma confidencial. Las fotografías que presentamos no permiten la identificación de las pacientes.

\section{RESULTADOS}

El grado de satisfacción fue bueno en 120 pacientes $(85.71 \%)$, regular en $10(7.15 \%)$ y

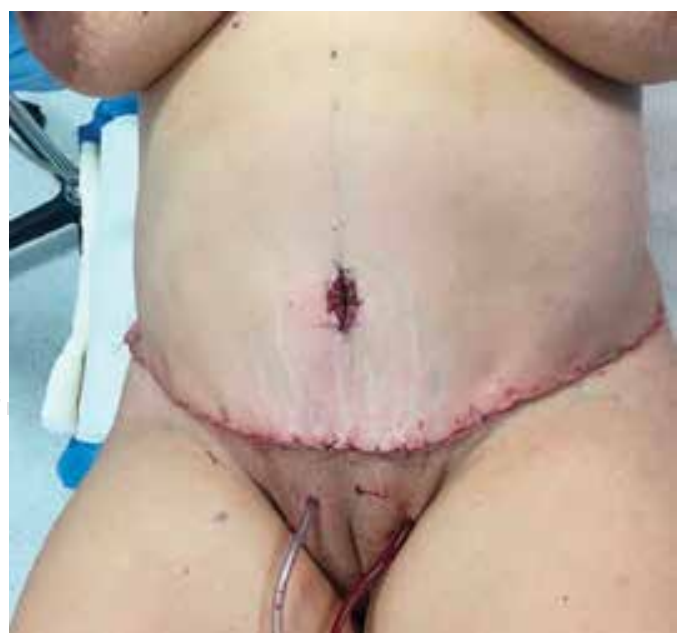

Figura 8: Cierre en tres planos. 
Figura 9:

Resultados de frente a los cuatro meses postquirúrgicos.

Figura 10:

Resultados de costado a los cuatro meses postquirúrgicos.
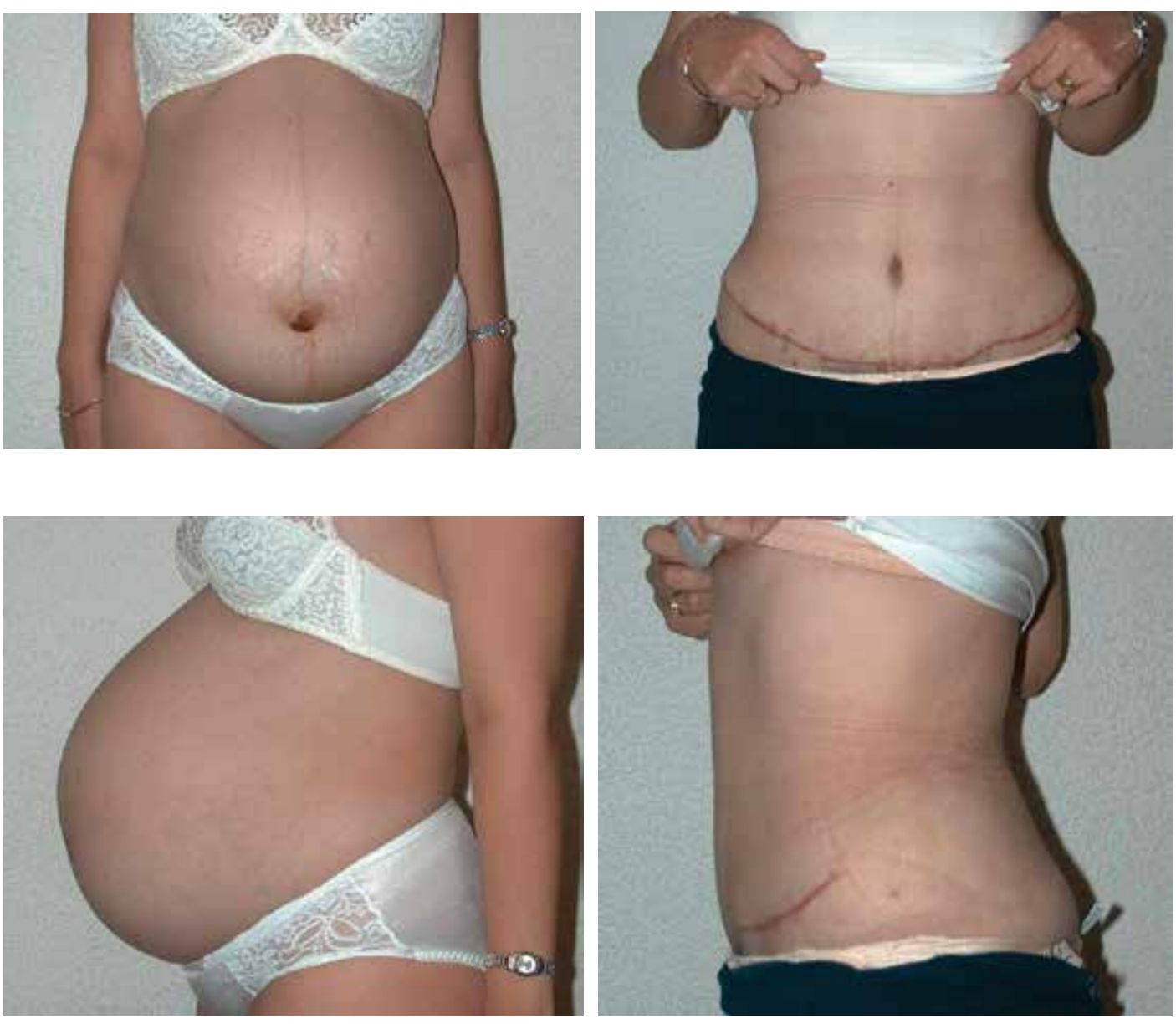

malo en 10 (7.15\%). El tiempo promedio de duración de la cirugía fue de tres horas (Figuras 9 a 11). La diástasis de los músculos rectos anteriores fue de 10 a $15 \mathrm{~cm}$ (promedio $12 \mathrm{~cm}$ ); la corrección de la diástasis fue satisfactoria en $100 \%$ de las pacientes. 10 pacientes $(7.15 \%)$ requirieron transfusión sanguínea. Realizamos la lipoaspiración de los costados en 130 pacientes $(92.85 \%)$ con resultados satisfactorios por mejora importante de la silueta de la cintura, con eliminación total de las estrías en 130 pacientes (92.85\%).

Las complicaciones encontradas en las 140 pacientes en 25 años fueron:

Menores en 10 pacientes (7.15\%). Presencia de seroma en 10 pacientes (7.15\%). Presencia de cicatrices hipertróficas y/o anchas en cuatro pacientes (28.57\%). También se incluyen estas cuatro pacientes que presentaron seroma.
Tres casos con dehiscencia de herida (2.14\%). Tres $(2.14 \%)$ con necrosis cutánea en forma triangular infraumbilical en un promedio de $8 \times 5 \mathrm{~cm}$ de dimensión, manejadas con curaciones, desbridamiento de tejido necrosado y cierre en una segunda intervención, con avance central de colgajos bilaterales dejando una cicatriz vertical del ombligo a la cicatriz inicial; apoyados con antibioticoterapia previo cultivo y antibiograma del área comprometida para determinar el antibiótico de elección (Figura 12).

No hemos tenido complicaciones mayores o severas como tromboembolia pulmonar, trombosis venosa profunda, coagulación intravascular diseminada, ni defunciones

El resultado de patología fue aumento del tamaño de los vasos sanguíneos y presencia de vasos de neoformación, así como cambios en la matriz cutánea (Figuras 13 y 14). 


\section{DISCUSIÓN}

La combinación de abdominoplastia más cesárea y en algunos casos salpingoclasia y lipoaspiración de los costados es un procedimiento solicitado por algunas pacientes que por su estilo de vida profesional y/o familiar las invita a solicitarla, ya que se realiza en un mismo periodo de convalecencia obligada por la cesárea, mencionando que al llevarse a cabo la paciente tendría el tiempo requerido para los cuidados maternales y la recuperación de la cirugía, evitando un segundo tiempo y riesgo quirúrgico. Son escasas las publicaciones que existen del tema y son controvertidas. Algunos autores consideran que es un procedimiento seguro y que se pueden obtener buenos resultados, mientras que otros opinan que es un procedimiento con alta incidencia de complicaciones incluyendo las mortales. ${ }^{14-18}$ Contamos con 25 años de experiencia con 140 pacientes operadas con un protocolo estricto de manejo perioperatorio. No hemos tenido las complicaciones mortales reportadas, las complicaciones han sido menores y los resultados en la mayoría de los casos han sido muy satisfactorios.

Dentro del protocolo de este procedimiento se hace una valoración de la madre y del producto. Elegimos pacientes en perfectas condiciones de salud, con control estricto perinatal y un alto grado de cooperación. La edad gestacional del producto debe ser de 36 a 41 semanas; no debe haber datos de malformaciones fetales o de riesgo de sufrimiento o muerte perinatal. El pilar más importante es el trabajo multidisciplinario en un equipo bien integrado con experiencia en el tema, preparado para identificar los riesgos y tratarlos en forma oportuna cuando lleguen a presentarse.

Recomendamos a la paciente y su pareja sexual aceptar una salpingoclasia bilateral para evitar embarazos futuros por el riesgo de alterar nuevamente los tejidos abdominales y su estética.

Los resultados estéticos no varían en forma importante en relación a las pacientes no
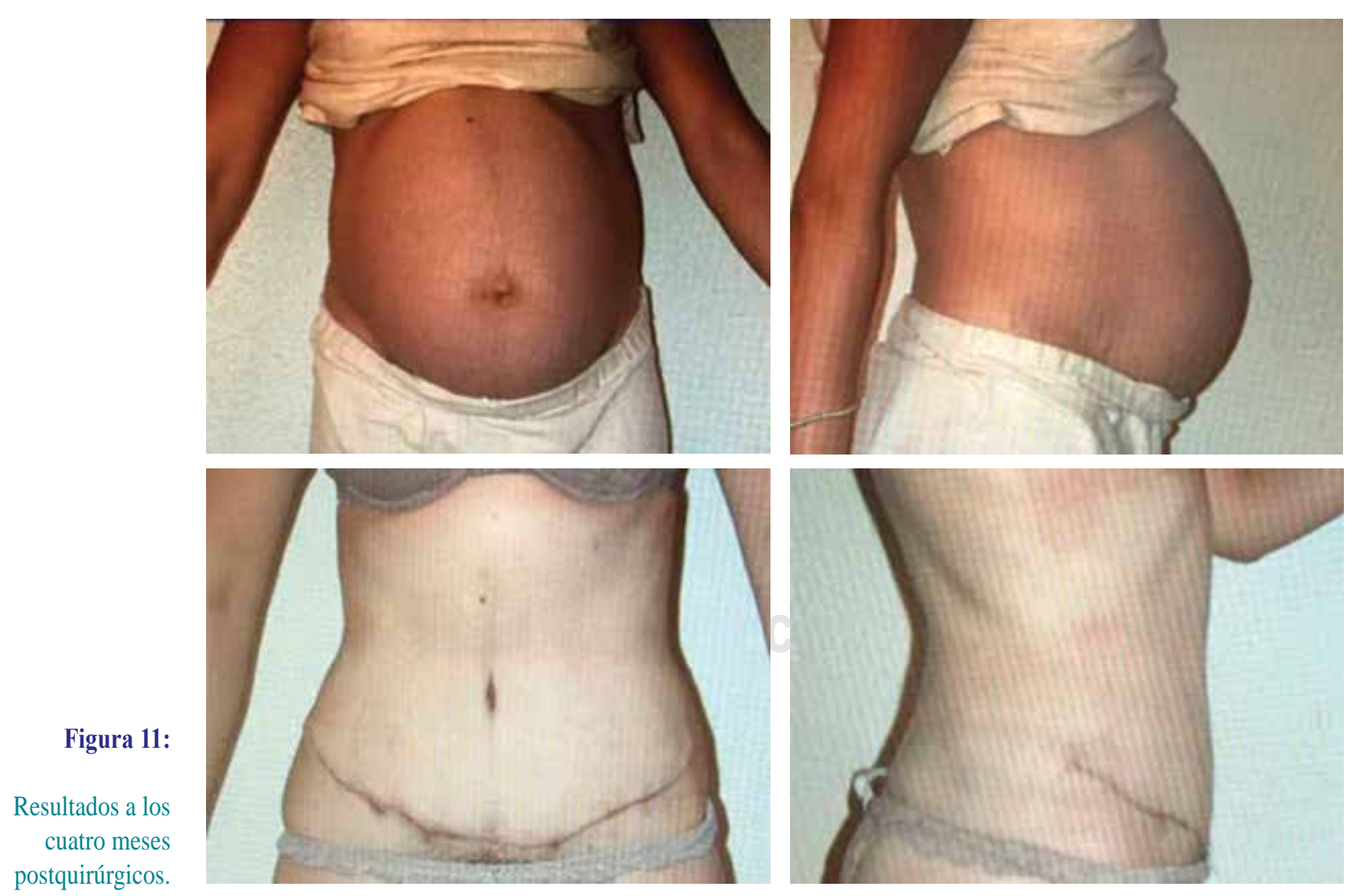

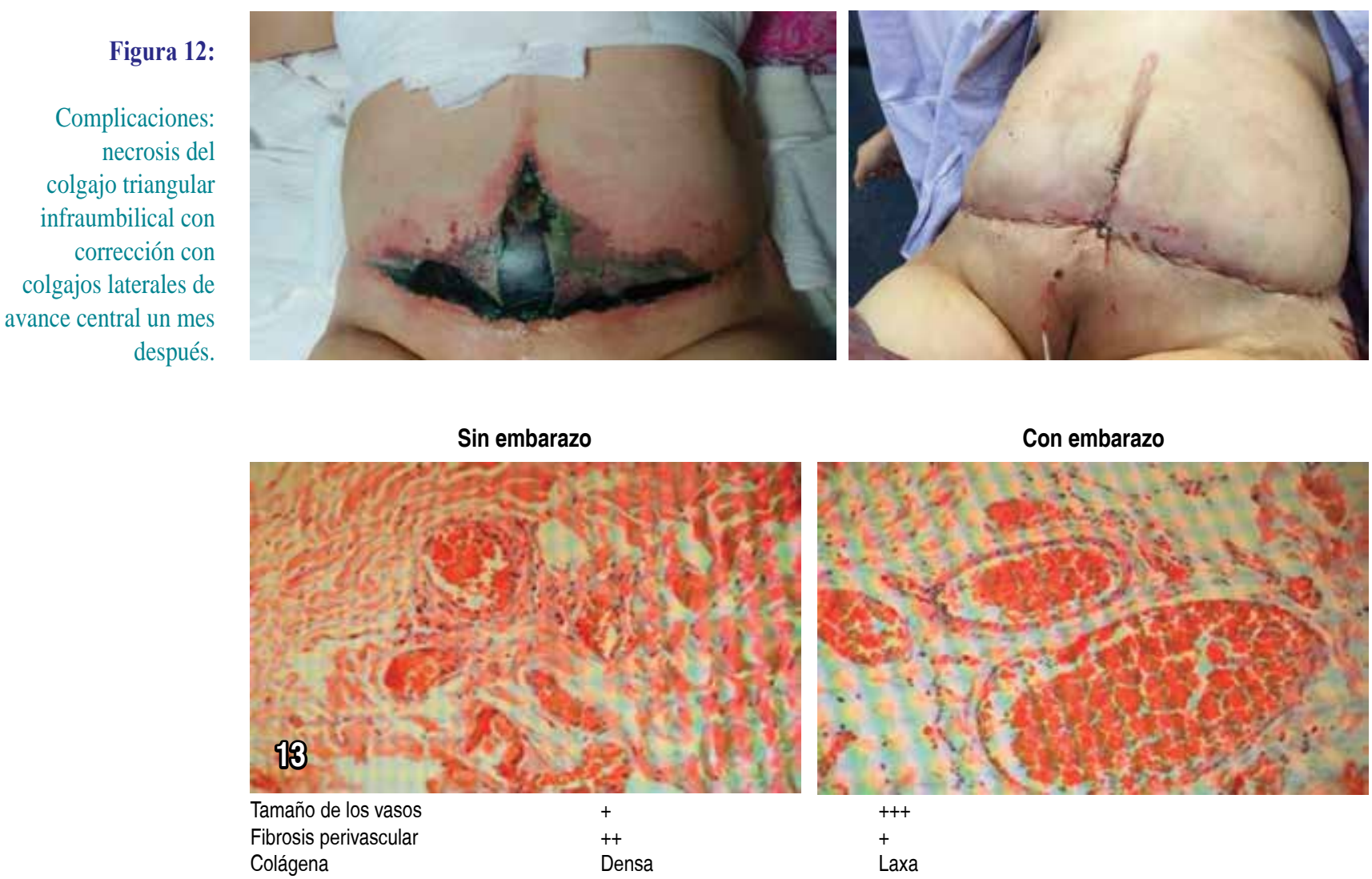

Figura 13 y 14:

Histopatología comparativa de tejidos de pacientes con y sin embarazo.

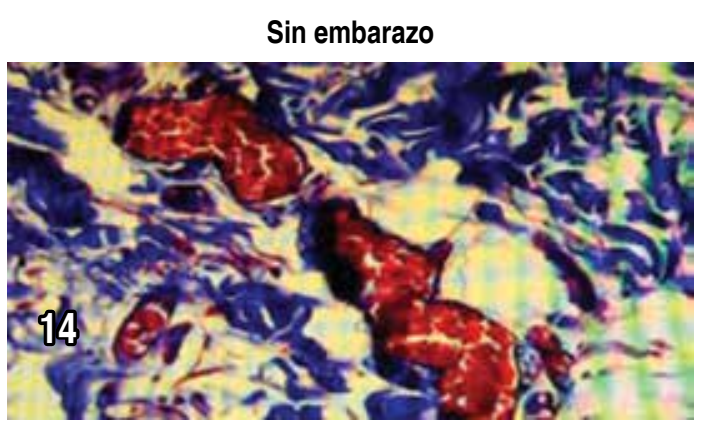

Tinción tricrómica de Masson (colágena) Disposición en haces Vasos colapsados

embrazadas. Asimismo, las complicaciones presentadas son similares en ambos tipos de pacientes. Cabe mencionar que los resultados postoperatorios inmediatos no son los ideales; sin embargo, a los cuatro meses, periodo de alta de la paciente en el que ya resuelta la involución uterina, la retracción cutánea del colgajo, la eliminación del edema y la retención de líquidos de la liposucción y otros factores propios del embarazo contribuyen

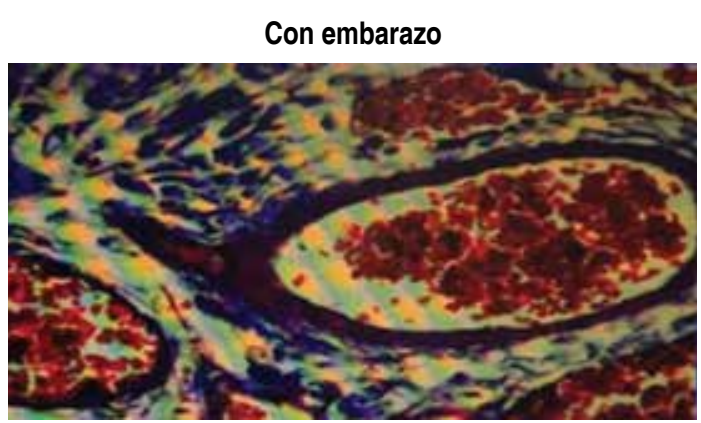

Delgados a una mejoría agregada de la estética de la pared abdominal.

Generalmente dentro del primer tercio de la vida de la mujer, justo en la mejor edad reproductiva y juventud, cursa con embarazos. $\mathrm{Si}$ la paciente no se mantiene en el peso ideal o incrementa su peso en más de $11 \mathrm{~kg}$ durante la gestación, los cambios cutáneos esperados en el embarazo se exacerban produciendo pérdida de la elasticidad de la piel, presencia 
de estrías cutáneas, elongación de la aponeurosis de los músculos rectos abdominales con el consiguiente adelgazamiento y diastasis. Estas alteraciones se asocian con frecuencia a hernia umbilical, adelgazamiento o desplazamiento de la grasa central, tan severa que se transparenta el útero. Los vasos sanguíneos aumentan en cantidad y tamaño, también se incrementa la colágena y se reorganizan los puentes de fibrina y elastina, cambios similares a los observados en la expansión tisular, por lo que se ha considerado que el embarazo es un tipo de expansor tisular natural. Algunos hallazgos histopatológicos observados en los tejidos de las pacientes con embarazo confirman el aumento del tamaño de los vasos sanguíneos y la presencia de vasos de neoformación, así como los cambios en la matriz cutánea. Los resultados de patología de los segmentos dermoadiposos que resecamos confirman estos hallazgos.

Hemos realizado esta combinación de procedimientos por más de 25 años, considerando que el embarazo es una expansión natural de los tejidos abdominales, que adquieren características idóneas de vascularización, producción y reorganización de los tejidos propios de la piel para un adecuado manejo durante la cirugía. Con la estrategia establecida hemos podido satisfacer la solicitud de las pacientes de llevar a cabo en forma simultánea los procedimientos con resultados satisfactorios y seguros, evitando en un futuro someterse a otra cirugía para corrección de los defectos de la pared abdominal secundarios al embarazo. Es importante señalar que la estética del abdomen mejora considerablemente después del cuarto mes, atribuible a la involución de los órganos y de la cubierta cutánea que retornan a sus condiciones naturales.

\section{CONCLUSIÓN}

Consideramos que la realización de la abdominoplastia-cesárea es un procedimiento que efectivamente no está en el tiempo y condiciones ideales para llevarse a cabo; sin embargo, de acuerdo a nuestra experiencia con 140 pacientes no encontramos limitantes para efectuarla cuando es solicitada por la paciente, pero es de suma importancia aplicar los criterios de inclusión y exclusión mencionados, así como llevar el protocolo descrito para que este procedimiento resulte en forma satisfactoria y segura.

\section{REFERENCIAS}

1. ISAPS international Survey on Aesthetic/Cosmetic Procedures Performed in 2017. Available in: http:// www.isaps.org/news/isaps-global-statistics.

2. Grazer FM, Jong RH. Fatal outcomes from liposuction. Census survey of cosmetic surgeons. Plast Reconstr Surg 2000; 105: 436-446.

3. Saad AN, Parina R, Chang D, Gosman AA. Risk of adverse outcomes when plastic surgery procedures are combined. Plast Reconstr Surg 2014; 134 (6): 1415-1422.

4. Rao RB, Ely SF, Hoffman RS. Deaths related to liposuction. N Engl J Med 1999; 340 (19): 14711475.

5. Ramírez-Guerrero JA. Liposucción. Consideraciones anestésicas y perioperatorias. Rev Mex Anest 2007; 30 (4): 233-241.

6. Grazer FM, Jong RH. Fatal outcomes from liposuction. Census survey of cosmetic surgeons. Plast Reconstr Surg 2000; 105: 436-446.

7. Winocour J, Gupta V, Ramírez JR, Shack RB, Grotting JC, Higdon KK. Abdominoplasty: risk factor, complications rates, and safety of combined procedures. Plast Reconstr Surg 2015; 136: 597e-606e.

8. Poore SO, Sillah N, Mahajan AY, Gotowski KA. Patient Safety in the Operating Room: II intraoperative and postoperative. Plast Reconstr Surg 2012; 130: 10481058.

9. Cárdenas SCM, Gutiérrez SJE, Márquez EC, Sánchez MFP, Gallardo PGJ, Eljure EM. Tromboprofilaxis en cirugía plástica: ¿cuándo, cómo y por qué? Cir Plast 2013; 23 (3): 168-178.

10. Lehnhardt M, Homann HH, Daigeler A, Hauser J, Palka P, Steinau HU. Major and lethal complication of liposuction. Plast Reconstr Surg 2008; 121 (6): 396e-403e.

11. Iglesias-Almanza NR, Guirola de la Parra J, PérezAssef H, Fernández-Gutiérrez R, Herrera-Collado R. Trastornos de la coagulación en el embarazo. Med Ciego [Revista en Internet]. 1996 Disponible en: http:// bvs.sld.cu/revistas/mciego/vol2_01_96/a8_v2_0196. html.

12. Ojeda-González JJ, Rodríguez-Álvarez M, EstepaPérez JL, Piña-Loyola CA, Cabeza-Poblet BL. Cambios fisiológicos durante el embarazo. Su importancia para el anestesiólogo. Medisur 2011; 9 (5): 484-491.

13. Emily J, Curtis KM, Gaffield ME. Risk of venous thromboembolism during the postpartum period: a systematic review. Obst Gynecol 2011; 117 (3): 691703.

14. Eli A, Essam A. Abdominoplasty combined with Cesarean delivery: evaluation of the practice. Aesth Plast Surg 2011; 35 (1): 80-86.

15. Thabet WN, Hossny AS, Sherif NA. Feasibility of abdominoplasty with Cesarean section. Inter J Woman's Health 2012; 4: 115-121. 
16. Benn TE, Spera CE. Abdominoplasty combined with cesarean section: discussion of the evidence. Am J Cosmetic Surg 2014; 31 (4): 260-263.

17. Matarasso A, Smith D M. Strategies for aesthetic reshaping of the postpartum patient. Plast Reconstr Surg 2015; 136 (2): 245-257.

18. Iribarren-Moreno R, Cuenca-Pardo J, Ramos-Gallardo G. Is plastic surgery combined with obstetrical procedures safe? Aesthet Plast Surg 2019; 43 (5):
1396-1399. https://doi.org/10.1007/s00266-01901448-9.

Correspondencia:

Dr. Jaime González-Mendoza

Spaplastic: Sierra de las Palomas Núm. 109, Bosques del Prado Sur, 20127

Aguascalientes, Aguascalientes, México.

E-mail: spaplastic@prodigy.net.mx 